\title{
Regular and Chaotic Motion in a Restricted Three-Body Problem of Astrophysical Interest
}

\author{
J. C. Muzzio, F. C. Wachlin and D. D. Carpintero \\ Facultad de Ciencias Astronómicas y Geofísicas de la Universidad \\ Nacional de La Plata and PROFOEG-CONICET, Argentina
}

\begin{abstract}
We have studied the motion of massless particles (stars) bound to a stellar system (a galactic satellite) that moves on a circular orbit in an external field (a galaxy). A large percentage of the stellar orbits turned out to be chaotic, contrary to what happens in the usual restricted three-body problem of celestial mechanics where most of the orbits are regular. The discrepancy is probably due to three facts: 1) Our study is not limited to orbits on the main planes of symmetry, but considers three-dimensional motion; 2) The force exerted by the satellite goes to zero (rather than to infinity) at the center of the satellite; 3) The potential of the satellite is triaxial, rather than spherical.
\end{abstract}

\section{Introduction}

Jefferys (1966) and Hénon (1966a, b) noticed the presence of chaotic (at that time called ergodic or semiergodic) orbits in the restricted three-body problem with two equal main masses. Later on, Hénon (1970) investigated Hill's case (one of the main masses vanishingly small) and found that "ergodic regions are very small and are not represented separately" (in his figure).

Chaotic orbits are more relevant in the elliptic case, as shown by Wintner and Murray (1996), which is reasonable because if the main masses do not move on circular orbits we lose Jacobi's integral.

Rix and White (1989) and Stewart (1993) investigated models more astrophysically oriented (two equal-mass galaxies on circular orbits). Rix and White found mainly regular orbits around each individual galaxy and chaotic motion for particles that moved from one galaxy to the other. Stewart, instead, not only found significant chaotic motion, but also Lyapunov times that were shorter than the age of the galaxies; nevertheless, he also found Lyapunov times longer than the age of the Universe for particles released deep inside the potential wells of the galaxies.

Carpintero et al. (1999) (hereafter, Paper I) considered a satellite moving on a circular orbit around a galaxy. Using different initial conditions (e.g., zero initial velocity, and initial positions on one of the main planes of symmetry with the initial velocity perpendicular to that plane) they obtained a variety of bound orbits that were classified using the frequency analysis code of Carpintero and Aguilar (1998); the Lyapunov characteristic exponents were also obtained for a sample of orbits. They found many chaotic orbits, even for energy values that 
kept the stars within the half-mass radius of the satellite. Moreover, they found Lyapunov times that were shorter than the age of the Universe.

The same frequency analysis of Paper I was applied to Heggie-Ramamani (1995) models of galactic satellites by Muzzio et al. (1999) (hereafter, Paper II). Since those models provide the distribution function, they were able to derive percentages of chaotic orbits, which turned out to be very high: between about $53 \%$ and $65 \%$, for models resembling globular clusters, and about $23 \%$ for a model resembling a dwarf galaxy.

Therefore, it is clear that chaotic motion is very significant inside galactic satellites. Now, the problem investigated in Papers I and II is essentially a threebody problem, albeit of astrophysical (rather than celestial mechanical) interest. Why are their results so different from those of Hénon? There are probably three main causes. First, Merritt and Fridman (1996) have pointed out that motion in the principal planes of symmetry is a poor guide to the three dimensional motion, and the studies of the classical problem were usually limited to orbits on the orbital plane of the two main masses, while our own studies included all kinds of orbits. Second, it is known that in a galactic satellite the force goes to zero at the center of the satellite, while the Newtonian force of the classical problem goes to infinity; as a result, in the latter case the motion near the secondary body is dominated by the Newtonian force, while in the innermost parts of a galactic satellite the attractive force of the satellite competes with the Coriolis force, resulting in chaotic motion (Paper II). Third, in the classical problem the main masses are spherical, while in the astrophysical problem triaxial satellites are used, and that triaxiality probably contributes to the inception of chaos.

Here we want to investigate again the astrophysical problem using spherical satellites to decide how important the triaxiality of the satellite is for the chaotic motion. Clearly, this is not a realistic problem since, as already indicated in Papers I and II (whose results may be relevant to those interested in realistic cases), a satellite stellar system cannot be spherical because, at the very least, it will be deformed by the tidal forces. Nevertheless, as our aim is to ascertain the importance of triaxiality on the emergence of chaos in the astrophysical threebody problem, the comparison of the previous more realistic results with those from the present purely theoretical case is most suitable for our purpose.

\section{Numerical experiments and results}

Just as we had done in Papers I and II, we considered satellites of unit mass moving on a circular orbit of 100 units radius with an angular velocity of 0.5 units in the field of a singular isothermal sphere (i.e., a logarithmic potential); the tidal radii of the satellites turn out to be about 1.26 . The difference with those investigations (where triaxial satellites were employed) is that, in the present case, the satellites are modelled with Plummer spheres, whose potential is:

$$
U(r)=-\frac{G M}{\sqrt{b^{2}+r^{2}}},
$$

where $G$ is the gravitational constant (taken as unit value), $M$ the satellite's mass, $r$ the radius and $b$ the softening parameter. We generated initial positions and velocities using the distribution function of the Plummer model (e.g., Binney 


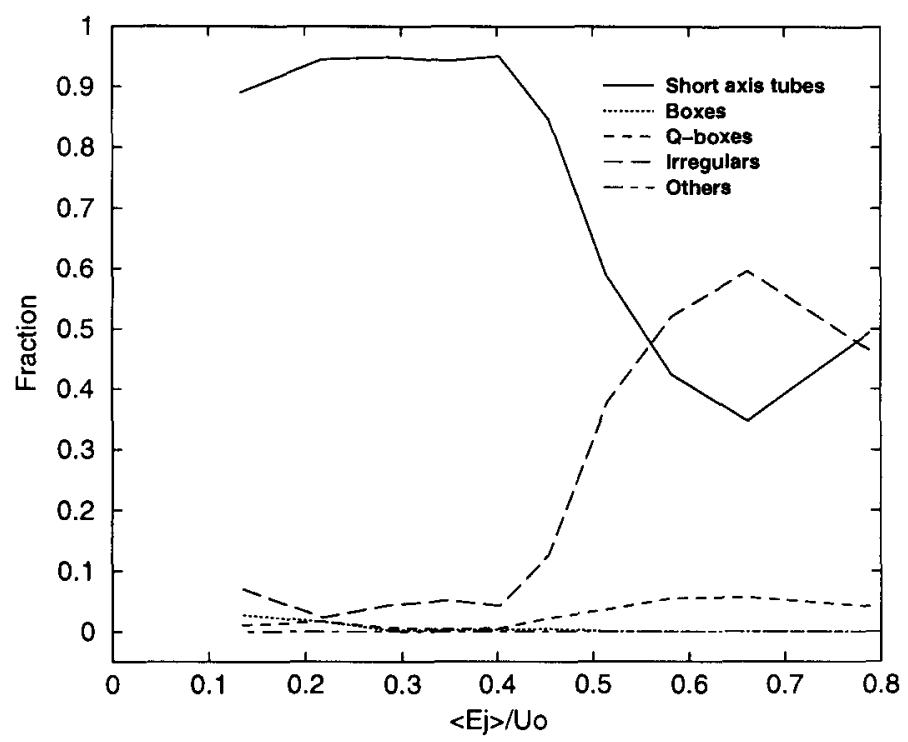

Figure 1. Fractions of the different orbital types vs. the orbital energy (normalized to the value of the central potential energy) for the $b=0.0458$ model.

and Tremaine, 1987) and a random number generator and, subsequently, we eliminated all those initial conditions that yielded positive values of the energy (actually, of the Jacobi integral) when the Plummer model was placed on its orbit in the galactic field. Two values of $b$ were adopted: 0.2290 (which yields a potential very similar to that of Paper I) and 0.0458 (one-fifth of the previous value, yielding a much more concentrated satellite); the half-mass radius that corresponds to the former is 0.299 , and that of the latter is 0.0597 .

A total of 5562 orbits with $b=0.229$, and 4992 with $b=0.0458$, were classified using the Carpintero and Aguilar (1998) code. Figure 1 gives the fractions of the different orbital types as a function of the Jacobi integral $\left(E_{j}\right)$ normalized to the value of the central potential $\left(U_{0}\right)$ when initial conditions are grouped into ten energy bins for the model with $b=0.0458$, and it can be compared to Figure 1 of Paper II. Similarly, Figure 2 gives the fraction of chaotic orbits for both models, again as a function of the normalized value of the Jacobi integral, and can be compared to Figure 2 of Paper II. The total fractions of chaotic orbits are 0.230 and 0.242 for $b=0.2290$ and $b=0.0458$, respectively.

\section{Discussion}

The fractions of chaotic orbits in the "globular cluster" models of Paper II were in the 0.53 to 0.65 range, that is, more than twice higher than the values obtained 


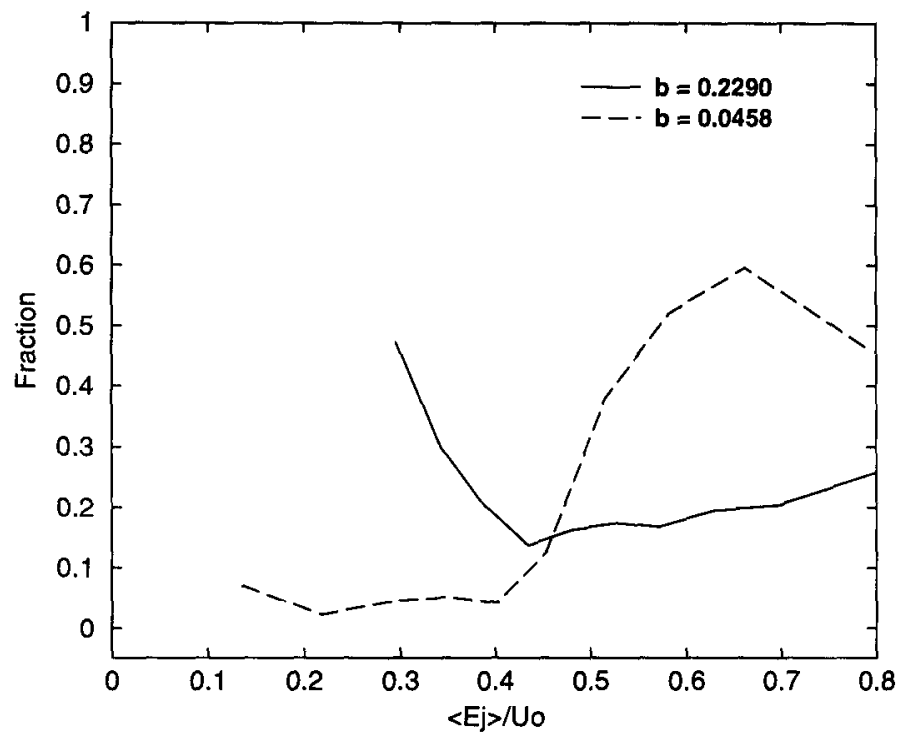

Figure 2. Fractions of chaotic orbits vs. the orbital energy (normalized to the central potential energy) for both models.

in the present investigation. Moreover, as already indicated in that paper, the more triaxial the model, the higher the chaoticity. Therefore, the low values obtained here for spherical models seem to confirm that triaxiality helped the inception of chaos in our astrophysical three-body problem. Nevertheless, as discussed in Paper II, and as shown by the present Figures 1 and 2, there is a complicated relationship between chaoticity and the orbital energy, so that it is worthwhile to examine in more detail what those figures tell us before accepting what the comparison of the global fractions of chaotic orbits suggests.

Our Figure 1 is strikingly similar to Figure 1 of Paper II, except for one fact: the present fractions of tube orbits are much higher (and, correspondingly, those of irregular orbits much lower) than those of our previous investigation. A comparison of our Figure 2 with Figure 2 of Paper II reveals that also for our $b=0.2290$ model the fractions of chaotic orbits are much lower than the corresponding values of the $W_{0}=2.5$ model of Paper II (the least concentrated one in that paper). In Paper II we explained the change in the fractions of chaotic orbits as we move through orbits of different energy (or Jacobi constant) by the interplay of three forces: the attractive force of the satellite, the Coriolis force and the tidal force (the asymmetry of Fig. 5 of Paper I provides further proof of the important role of the Coriolis force on chaoticity). We also indicated that triaxiality probably enhanced chaoticity, particularly in the model with $W_{0}=2.5$ (the most triaxial of those considered in Paper II). Therefore, the great similarity between the results of the $W_{0}=6.0$ model of Paper II and the present $b=0.0458$ model is most likely due to the fact that the same interplay of forces is at work 
in both cases; alternatively, the lower chaoticity of the Plummer model is in all likelihood due to its spherical symmetry. A similar story is told by Figure 2 . Although the trend of the results of our $b=0.2290$ model differs somewhat from those of the $W_{0}=2.5$ model of Paper II, the difference is probably due to the fact that the present model has a larger fraction of stars far from the center of the satellite than the Heggie-Ramamani model of our previous work, causing the increase of chaoticity towards the low $\left\langle E_{j}\right\rangle / U_{0}$ values. Nevertheless, it is perfectly clear that the present, spherical, model displays much lower fractions of chaotic orbits than the triaxial models of our previous work.

Alternatively, it is also clear that even the present spherical models display a fairly high level of chaoticity, as compared with the results of the classical three-body problem. Therefore, as surmised in our previous work, we may conclude that both triaxiality and the vanishingly small attractive force of the satellite as we approach its center, are the main physical causes of the different chaoticity found in the classical and in the astrophysical three-body problem. A third cause is probably just a matter of sampling: while we investigated a wide variety of orbits, classical studies concentrated in orbits on the plane of symmetry, where the motion may be mainly regular.

Acknowledgments. This investigation was supported by grants from the Universidad Nacional de La Plata and the Consejo Nacional de Investigaciones Científicas y Técnicas de la República Argentina. The assistance of the International Astronomical Union to JCM, through a travel grant, is gratefully acknowledged.

\section{References}

Binney, J. \& Tremaine, S. 1987, Galactic Dynamics, Princeton: Princeton University Press

Carpintero, D. D., \& Aguilar, L. A. 1998, MNRAS, 298, 1

Carpintero, D. D., Muzzio, J. C. \& Wachlin, F. C. 1999, Cel. Mech. \& Dynam. Astron., in press

Heggie, D. C., \& Ramamani, N. 1995, MNRAS, 272, 317

Hénon, M. 1966a, Bull. Astron. 3e. ser. T. 1, Fasc. 1, 57

Hénon, M. 1966b, Bull. Astron. 3e. ser. T. 1, Fasc. 2, 49

Jefferys, W. H. 1966, AJ, 71, 306

Merritt, D. \& Fridman, T. 1996, ApJ, 460, 136

Muzzio, J. C., Wachlin, F. C. \& Carpintero, D. D. 1999, in World Scientific Advanced Series in Astrophysics and Cosmology, V. G. Gurzadyan \& R. Ruffini, in press

Rix H.-W. R., \& White, S. D. M. 1989, MNRAS, 240, 941

Stewart, P. 1993, A\&A, 269, 135

Wintner, O. C. \& Murray, C. D. 1996, in Chaos in Gravitational $N$-Body Systems, J. C. Muzzio, S. Ferraz-Mello \& J. Henrard, Dordrecht: Kluwer, 215 